\title{
Analysis of Soret and Dufour Effects on Unsteady MHD Convective Flow past a Semi-Infinite Vertical Porous Plate via Finite Difference Method
}

\author{
Deepa Gadipally ${ }^{*}$, Murali Gundagani² \\ ${ }^{1}$ Department of Mathematics, Chaitanya Bharathi Institiute of Technology, India. \\ ${ }^{2}$ Department of Mathematics, School of Technology, GITAM, India. \\ * Corresponding author. email: deepagadipally@yahoo.com \\ Manuscript submitted June 10, 2014; accepted September 10, 2014. \\ doi: 10.7763/IJAPM.2014.V4.306
}

\begin{abstract}
An analysis is made for the unsteady magnetohydrodynamic (MHD) free convection flow of a viscous, incompressible, electrically conducting fluid past a semi infinite vertical porous moving plate taking in to account the mass transfer with variable viscosity and thermal conductivity. An implicit finite difference method is used to solve the unsteady, non linear and coupled governing equations. A parametric study illustrating the influence of different flow parameters on velocity, temperature and concentration are investigated. Numerical results are presented for various parameters.
\end{abstract}

Key words: MHD, dufour, soret, variable viscosity and thermal conductivity.

\section{Introduction}

The Numerical methods are of great importance for approximating the solutions of nonlinear partial differential equations, especially when the nonlinear differential equation under consideration faces difficulties in obtaining its exact solution. In this latter case, we usually resort to one of the efficient numerical methods. The advanced numerical methods and computer technology have shown the way in which such difficult problems can be solved. Finite difference methods play a major role in solving partial differential equations. Coupled heat and mass transfer phenomenon in porous media is gaining attention due to its interesting applications. The flow phenomenon in this case is relatively more complex than that in the pure thermal/solutal convection process. Processes involving heat and mass transfer in porous media are often encountered in the chemical industry, in reservoir engineering in connection with thermal recovery process, and in the study of the dynamics of hot and salty springs of a sea. Combined buoyancy generated heat and mass transfer due to temperature and concentration variations, in fluid-saturated porous media have several important applications in a variety of engineering processes including heat exchanger devices, petroleum reservoirs, chemical catalytic reactors, solar energy porous wafer collector systems, ceramic materials has been studied by Kaviancy et al. [1], migration of moisture through air contained in fibrous insulations and grain storage installations, and the dispersion of chemical contaminants through water-saturated soil, superconvecting geothermics etc are investigated by Dunn, J. C. and Hardee, H. C. [2]. The vertical free convection boundary layer flow in porous media owing to combined heat and mass transfer has been investigated by Nield, D. A. [3] and Khair [4]. Lai and Kulacki [5] 
used the series expansion method to investigate coupled heat and mass transfer in natural convection from a sphere in a porous medium. Comprehensive reviews of porous media thermal/species convection have been presented by Nield and Bejan [3], vafai [6] and Pop and Ingham [7].

Raptis [8] analyzed hydromagnetic free convection flow through a porous medium between two parallel plates. Gribben [9] studied boundary layer flow over a semi-infinite plate with an aligned magnetic field in the presence of a pressure gradient. He obtained solutions for large and small magnetic Prandtl numbers using the method of matched asymptotic expansion. Raptis [10] studied mathematically the case of time varying two dimensional natural convective flow of an incompressible, electrically conducting fluid along an infinite vertical porous plate embedded in a porous medium. Helmy [11] studied MHD unsteady free convection flow past a vertical porous plate embedded in a porous medium. Kim [12] investigated unsteady MHD convective heat transfer past a semi-infinite vertical porous moving plate with variable suction by assuming that the free stream velocity follows the exponentially increasing small perturbation law.

All the above studies were confined to a fluid with constant viscosity. For most realistic fluids, the viscosity shows a rather pronounced variation with temperature. However, it is known that this physical property may change significantly with temperature. Then it is necessary to take into account the variation of viscosity with temperature in order to accurately predict heat transfer rates. Hossain and Munir [13] analyzed a two-dimensional mixed convection flow of a viscous incompressible fluid of temperature dependent viscosity past a vertical plate. Hossain et al. [14] discussed the effect variable viscosity on free convective flow past a vertical plate.

In all these studies Soret and Dufour effects are assumed to be negligible. Such effects are significant when density differences exist in the flow regime. For example when species are introduced at a surface in fluid domain, with different (lower) density than the surrounding fluid, both Soret and Dufour effects can be significant. Also, when heat and mass transfer occur simultaneously in a moving fluid, the relations between the fluxes and the driving potentials are of more intricate nature. It has been found that an energy flux can be generated not only by temperature gradients but by composition gradients as well. The energy flux caused by a composition gradient is called the Dufour or diffusion -thermo effect. On the other hand, mass fluxes can also be created by temperature gradients and this is called the Soret or thermal-diffusion effect. The thermal-diffusion (Soret) effect, for instance, has been utilized for isotope separation, and in mixture between gases with very light molecular weight $\left(\mathrm{H}_{2}, \mathrm{He}\right)$ and of medium molecular weight $\left(\mathrm{N}_{2}\right.$, air $)$, the diffusion -thermo (Dufour) effect was found to be of a considerable magnitude such that it cannot be ignored was studied by Eckert and Drake [15]. In view of the importance of this diffusion-thermo effect, Jha and Singh [16] studied the free-convection and mass transfer flow about an infinite vertical flat plate moving impulsively in its own plane, taking into account the Soret effects. Kishan et al. [17] studied the effects of viscous dissipation on free convection heat and mass transfer characteristics in a Darcian porous medium with Soret and Dufour effects. Recently, Gnaneswara Reddy and Bhaskar Reddy [18] studied the effects of variable viscosity and thermal conductivity on an unsteady two-dimensional laminar flow of a viscous incompressible electrically conducting fluid past a semi-infinite vertical porous moving plate taking into account the mass transfer.

However, the study of Soret and Dufour effects on unsteady MHD free convection flow of a dissipation fluid has received a little attention. Hence, the objective of the present paper is to analyze the Soret and Dufour effects on unsteady MHD mixed convective boundary layer flow of a viscous incompressible, electrically conducting fluid past a semi-infinite vertical porous moving plate taking into account the mass transfer with variable viscosity and thermal conductivity. The governing equations are transformed by using similarity transformation and the resultant dimensionless equations are solved by using the implicit finite difference scheme with the help of C-programming. 


\section{Formulation of the Problem}

An unsteady two-dimensional laminar mixed convective boundary layer flow of a viscous, incompressible, electrically conducting fluid past a semi-infinite vertical permeable moving plate, embedded in a uniform porous medium and subjected to a uniform transverse magneticfield, in the presence of thermal and concentration buoyancy effects, the temperature dependent viscosity and thermal conductivity is considered. The $\mathrm{x}^{\prime}$ - axis is taken along the vertical plate and the $\mathrm{y}^{\prime}$ - axis normal to the plate. It is assumed that there is no applied voltage, which implies the absence of an electric field. The transverse applied magnetic field and magnetic Reynolds number are assumed to be very small so that the induced magnetic field. We assume that the fluid properties are isotropic and constant, except for the fluid viscosity $\mu$, which is assumed to vary as an

$$
\frac{1}{\mu}=E\left(T^{\prime}-T_{r}^{\prime}\right)
$$

where

$$
E=\frac{\delta}{\mu_{\infty}} \text { and } \quad T_{r}^{\prime}=T_{\infty}^{\prime}-\frac{1}{\delta}
$$

Here $\mu_{\infty}$ and $\mathrm{T}^{\prime}{ }_{\infty}$ are the fluid free stream dynamic viscosity and the fluid free stream temperature $E$ and $T_{r}$ are the constants and their values depend on the reference state and thermal property of the fluid , i.e., $\delta$. In general, $E<0$ for fluid such as liquids and $E>0$ for gases. Also, we assume that the fluid thermal conductivity, $\alpha$, is assumed to vary as a linear function of temperature in the form

$$
\alpha=\alpha_{0}\left(1+a\left(T^{\prime}-T_{\infty}^{\prime}\right)\right)
$$

where $\alpha_{0}$ is the thermal diffusivity at temperature $T_{w}^{\prime}$ and $a$ is a constant depending on the nature of the fluid. In general $a>0$ for fluids such as water air, while $a<0$ for fluids such as lubrication oils. This form can be rewritten in the non-dimensional form:

$$
\alpha=\alpha_{0}(1+\beta \theta)
$$

where $\beta=a\left(T_{w}^{\prime}-T_{\infty}^{\prime}\right)$ is the thermal conductivity parameter and $T_{w}^{\prime}$ is the value of the plate temperature. The range of variations of $\beta$ can be taken as for air $0 \leq \beta \leq 6$, for water $0 \leq \beta \leq 0.12$ and for lubrications oils $-0.1 \leq \beta \leq 0$. Further due to the semi-infinite plane surface assumption, the flow variables are functions of normal distance $y^{\prime}$ and $t^{\prime}$ only. Now, under the usual Boussinesq's approximation, the governing boundary layer equations of the problem are

$$
\begin{gathered}
\frac{\partial v^{\prime}}{\partial y^{\prime}}=0 \\
\rho\left(\frac{\partial u^{\prime}}{\partial t^{\prime}}+v^{\prime} \frac{\partial u^{\prime}}{\partial y^{\prime}}\right)=-\frac{\partial p^{\prime}}{\partial x^{\prime}}+\frac{\partial}{\partial y^{\prime}}\left(\mu \frac{\partial u^{\prime}}{\partial y^{\prime}}\right)+\rho g \beta\left(T^{\prime}-T_{\infty}^{\prime}\right) \\
+\rho g \beta^{*}\left(C^{\prime}-C_{\infty}^{\prime}\right)-\frac{v \rho u^{\prime}}{K^{\prime}}-\sigma B_{0}^{2} u^{\prime}
\end{gathered}
$$




$$
\begin{gathered}
\frac{\partial T^{\prime}}{\partial t^{\prime}}+v^{\prime} \frac{\partial T^{\prime}}{\partial y^{\prime}}=\frac{\partial}{\partial y^{\prime}}\left(\alpha \frac{\partial T^{\prime}}{\partial y^{\prime}}\right)+\frac{D_{m} K_{T}}{C_{s} C_{p}} \frac{\partial^{2} C^{\prime}}{\partial y^{\prime 2}} \\
\frac{\partial C^{\prime}}{\partial t^{\prime}}+v^{\prime} \frac{\partial C^{\prime}}{\partial y^{\prime}}=D \frac{\partial^{2} C^{\prime}}{\partial y^{\prime 2}}+\frac{D_{m} K_{T}}{T_{m}} \frac{\partial^{2} T^{\prime}}{\partial y^{\prime 2}}
\end{gathered}
$$

Where $u^{\prime}, v^{\prime}$ are the velocity components in $x^{\prime}, y^{\prime}$ directions, $t^{\prime}$ the time, $p^{\prime}$ the pressure, $\rho$ the fluid density, $g$ the acceleration due to gravity, $\beta$ and $\beta^{*}$ the thermal and concentration expansion coefficients, $K^{\prime}$ the permeability of the porous medium, $T^{\prime}$ the temperature of the fluid in the boundary layer, $v$ the kinematic viscosity, $\sigma$ the electrical conductivity of the fluid, $T_{\infty}$ the temperature of the fluid far away from the plate, $C^{\prime}$ the species concentration in the boundary layer, $C_{\infty}^{\prime}$ the species concentration in the fluid far away from the plate, $B_{0}$ the magnetic induction, $\mu$ the fluid viscosity, $\alpha$ the fluid thermal diffusivity.

It is assumed that the permeable plate moves with a constant velocity in the direction of fluid flow and the free stream velocity follows the exponentially increasing small perturbation law. In addition, it is assumed that the temperature and the concentration at the wall as well as the suction velocity are exponentially varying with time.

The boundary conditions for the velocity, temperature and concentration fields are

$$
\begin{gathered}
u^{\prime}=u_{p}^{\prime}, \quad T^{\prime}=T_{\infty}^{\prime}+\varepsilon\left(T_{w}^{\prime}-T_{\infty}^{\prime}\right) e^{n^{\prime \prime}}, C^{\prime}=C_{\infty}^{\prime}+\varepsilon\left(C_{w}^{\prime}-C_{\infty}^{\prime}\right) e^{n^{\prime \prime} t^{\prime}} \text { aty }=0 \\
u^{\prime}=U_{\infty}^{\prime}=U_{0}\left(1+\varepsilon e^{n^{\prime} t^{\prime}}\right) T^{\prime} \rightarrow T_{\infty}^{\prime}, C^{\prime} \rightarrow C_{\infty}^{\prime} \text { aty } \rightarrow \infty
\end{gathered}
$$

where $u_{p}^{\prime}$, is the plate velocity, $T_{w}^{\prime}$ and $C_{w}^{\prime}$ the temperature and concentration of the wall respectively, $U_{\infty}^{\prime}$ - the free stream velocity, and $U_{0}$ and $\mathrm{n}^{\prime}$ - the constants. From Equation (1), it is clear that the suction velocity at the plate is either a constant or a function of time. Hence the suction velocity normal to the plate is assumed in the form

$$
v^{\prime}=-V_{0}\left(1+\varepsilon A e^{n^{\prime \prime}}\right)
$$

where $A$ is a real positive constant, $\varepsilon$ and $\varepsilon A$ are small values less than unity, and $V_{0}$ is scale of suction velocity which is non-zero positive constant. The negative sign indicates that the suction is towards the plate.

Outside the boundary layer, Equation (2) gives

$$
-\frac{1}{\rho} \frac{\partial p^{\prime}}{\partial x^{\prime}}=\frac{d U_{\infty}^{\prime}}{d t^{\prime}}+\frac{v}{k^{\prime}} U_{\infty}^{\prime}+\frac{\sigma}{\rho} B_{0}^{2} U_{\infty}^{\prime}
$$

In order to write the governing equations and the boundary conditions in dimensionless form, the 
following non-dimensional quantities are introduced.

$$
\begin{gathered}
u=\frac{u^{\prime}}{U_{0}}, v=\frac{v^{\prime}}{V_{0}}, y=\frac{V_{0} y^{\prime}}{v}, U_{\infty}=\frac{U_{\infty}^{\prime}}{U_{0}}, U_{p}=\frac{U_{p}^{\prime}}{U_{0}}, t=\frac{V_{0}^{2} t^{\prime}}{v} \\
\theta=\frac{T^{\prime}-T_{\infty}^{\prime}}{T_{w}^{\prime}-T_{\infty}^{\prime}}, \theta_{r}=\frac{T_{r}^{\prime}-T_{\infty}^{\prime}}{T_{w}^{\prime}-T_{\infty}^{\prime}}, C=\frac{C^{\prime}-C_{\infty}^{\prime}}{C_{w}^{\prime}-C_{\infty}^{\prime}}, n=\frac{n^{\prime} v}{V_{0}^{2}} \\
K=\frac{K^{\prime} V_{0}^{2}}{v^{2}}, \operatorname{Pr}=\frac{v}{\alpha_{0}}, S c=\frac{v}{D}, G r=\frac{v \beta g\left(T_{w}^{\prime}-T_{\infty}^{\prime}\right)}{U_{0}^{2} V_{0}^{2}} \\
G m=\frac{v \beta^{*} g\left(C_{w}^{\prime}-C_{\infty}^{\prime}\right)}{U_{0}^{2} V_{0}^{2}}, M=\frac{\sigma B_{0}^{2} v}{\rho V_{0}^{2}}
\end{gathered}
$$

In view of Equations (6) and (7), Equations (2) - (4) reduce to the following dimensionless form.

$$
\begin{aligned}
& \frac{\partial u}{\partial t}-\left(1+\varepsilon A e^{n t}\right) \frac{\partial u}{\partial y}=\frac{d U_{\infty}}{d t}+\frac{\partial^{2} u}{\partial y^{2}}+G r \theta \\
& +G m C+N \\
& \frac{\partial \theta}{\partial t}-\left(1+\varepsilon A e^{n t}\right) \frac{\partial \theta}{\partial y}=\frac{1}{\operatorname{Pr}}\left[(1+\beta \theta) \frac{\partial^{2} \theta}{\partial y^{2}}\right] \\
& +\frac{1}{\operatorname{Pr}} \beta\left(\frac{\partial \theta}{\partial y}\right)^{2} \\
& \frac{\partial C}{\partial t}-\left(1+\varepsilon A e^{n t}\right) \frac{\partial C}{\partial y}=\frac{1}{S c} \frac{\partial^{2} C}{\partial y^{2}}-S r \frac{\partial^{2} \theta}{\partial y^{2}}
\end{aligned}
$$

where $N=M+(1 / K)$ and $G r, G m, \operatorname{Pr}, \theta_{r}, \beta$ and $S c$ are the thermal Grashof number, solutal Grashof Number, Prandtl Number, variable viscosity parameter, thermal conductivity parameter and Schmidt number respectively.

The corresponding boundary conditions are

$$
\begin{gathered}
u=u_{p}, \quad \theta=1+\varepsilon e^{n t}, C=1+\varepsilon e^{n t} \text { at } y=0 \\
u \rightarrow U_{\infty}=1+\varepsilon e^{n t}, \theta \rightarrow 0, C \rightarrow 0 \text { at } y \rightarrow \infty
\end{gathered}
$$

\section{Solution of the Problem}

The Equations (9) - (11) are coupled, non-linear partial differential equations and these cannot be solved 
in closed-form. However, these equations are solved by using the implicit finite difference method. This reduced to the following form

$$
\begin{gathered}
a_{2}[i] u_{i+1}^{n+1}+b_{2}[i] u_{i}^{n+1}+c_{2}[i] u_{i-1}^{n+1}=d_{2}[i](12) a_{1}[i] \theta_{i-1}^{n+1}+b_{1}[i] \theta_{i}^{n+1}+c_{1}[i] \theta_{i-1}^{n+1}=d_{1}[i] \\
a C_{i-1}^{n+1}+b C_{i}^{n+1}+c C_{i-1}^{n+1}=d[i]
\end{gathered}
$$

where

$$
\begin{gathered}
A_{1}=1+\varepsilon e^{n t} \\
B_{1}[i]=\frac{\theta^{\prime}}{\theta-\theta_{r}} \\
a_{2}[i]=-\frac{k}{2 h}\left(A_{1}-B_{1}[i]\right)-\frac{r}{2} \\
d_{2}[i]=k\left(\varepsilon n e^{n t}+G r \theta+G m C+N U_{\infty}\right)+u_{i}^{n} \\
c_{2}[i]=\frac{k}{2 h}\left(A_{1}-B_{1}[i]\right)-\frac{r}{2} \\
a_{1}[i]=\frac{k A_{1} \operatorname{Pr}}{2 h}-r\left(1+t_{1}[i] \beta\right) \\
b_{1}[i]=\operatorname{Pr}+2 r\left(1+t_{1}[i] \beta\right)-k \beta \theta_{2}[i] \\
c_{1}[i]=-\frac{2 S A_{1} \operatorname{Pr}}{2 h}-r\left(1+t_{1}[i] \beta\right) \\
h=-r, b=2(S c+r)+\frac{2 S c A_{1} k}{h} \\
\left.c_{1} k i\right]=2 S c C[i]+k S r T_{2}[i]
\end{gathered}
$$

The skin-friction, Nusselt number and Sherwood number are important physical parameters for this type of boundary layer flow.

The skin-friction at the plate can be obtained, which is in non-dimensional form is given by

$$
C_{f}=\frac{\tau_{w}^{\prime}}{\rho U_{0} V_{0}}=\left(\frac{\partial u}{\partial y}\right)_{y=0}
$$


The rate of heat transfer coefficient can be obtained, which in the non-dimensional form, in terms of the Nusselt number, is given by

$$
N u=-x \frac{\left(\frac{\partial T}{\partial y^{\prime}}\right)_{y^{\prime}=0}}{T_{w}^{\prime}-T_{\infty}^{\prime}} \Rightarrow N u \mathrm{Re}_{x}^{-1}=-\left(\frac{\partial \theta}{\partial y}\right)_{y=0}
$$

where $\operatorname{Re} x=\frac{V_{0} x}{v}$ is the local Reynolds number.

The rate of mass transfer coefficient can be obtained, which in the non-dimensional form, in terms of the Sherwood number, is given by

$$
N u=-x \frac{\left(\frac{\partial T}{\partial y^{\prime}}\right)_{y^{\prime}=0}}{C_{w}^{\prime}-C_{\infty}^{\prime}} \Rightarrow S h \operatorname{Re}_{x}^{-1}=-\left(\frac{\partial C}{\partial y}\right)_{y=0}
$$

\section{Results and Discussion}

A representative set of numerical results is shown graphically in figures 1-10, to illustrate the influence of physical parameters thermal Grashof number $G r$, solutal Grashof number Gm, magnetic field parameter $M$, porous medium parameter $K$, variable viscosity parameter $\theta_{r}$, thermal conductivity parameter $\beta$, Schmidt number $S c$, Dufour number $D u$ and the Soret number $S r$ on the velocity, temperature and concentration profiles. In the numerical computation, the prandtl number $P r=0.71$ which corresponds to air and various values of the material parameters are used. In addition, the boundary condition $y \rightarrow \infty$ is approximate $y$ $\max =6$, which is sufficiently large for the velocity to approach the relevant stream velocity.

The effect of the thermal Grashof number $\mathrm{Gr}$ and the solutal grashof number $\mathrm{Gm}$ on the velocity profiles is presented in Fig. 1. It is observed that an increase in $G r$ leads to a rise in the values of velocity due to enhancement in buoyancy force. Here the positive value of $\mathrm{Gr}$ corresponds to cooling of the surface. It is also observed that the influence of solutal Grashof number $\mathrm{Gm}$ is to increase the velocity of the fluid indicating that species buoyancy as an accelerating affect on the flow field. It is evident from the figure that velocity distribution attains a distinctive maximum value in the vicinity of the plate surface and then decreases properly to approach the free stream value.

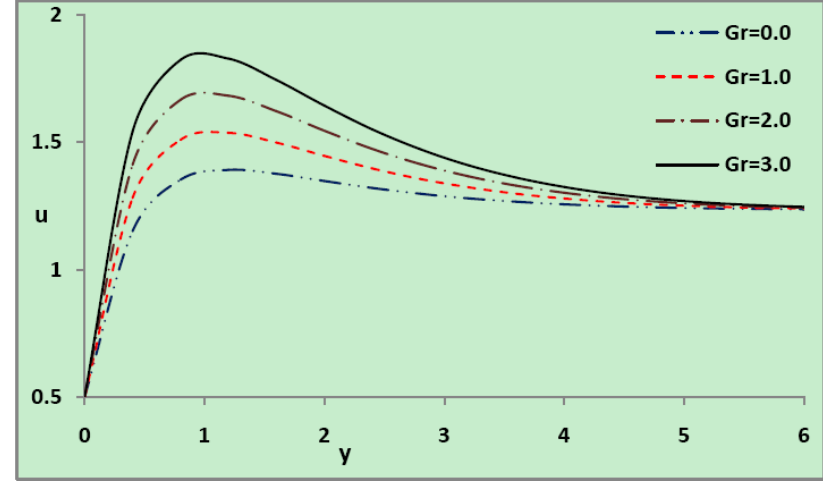

a)

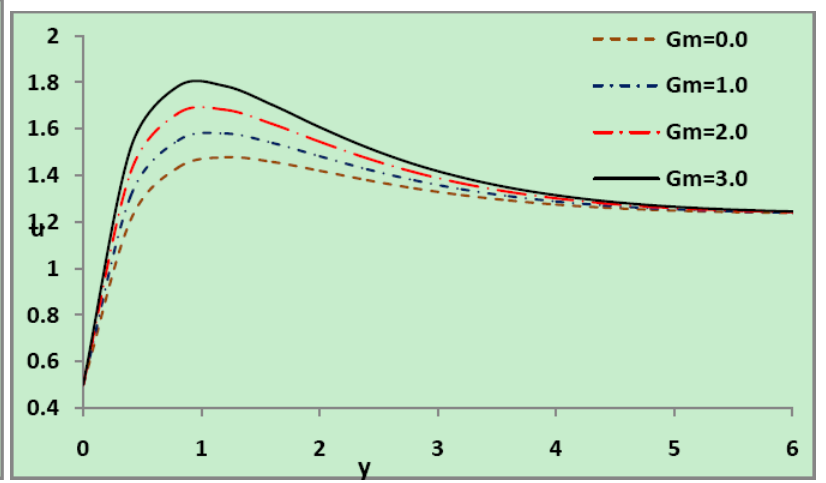

b)

Fig. 1 a). Velocity profiles for different values of $\mathrm{Gr}$; b) Velocity profiles for different values of $\mathrm{Gm}$. 


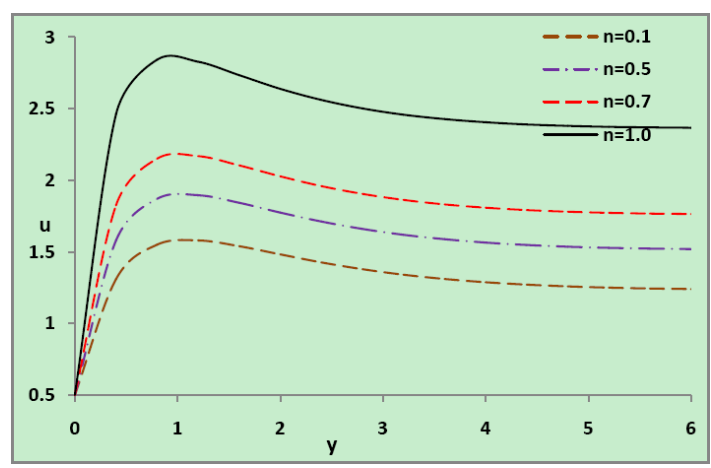

a)

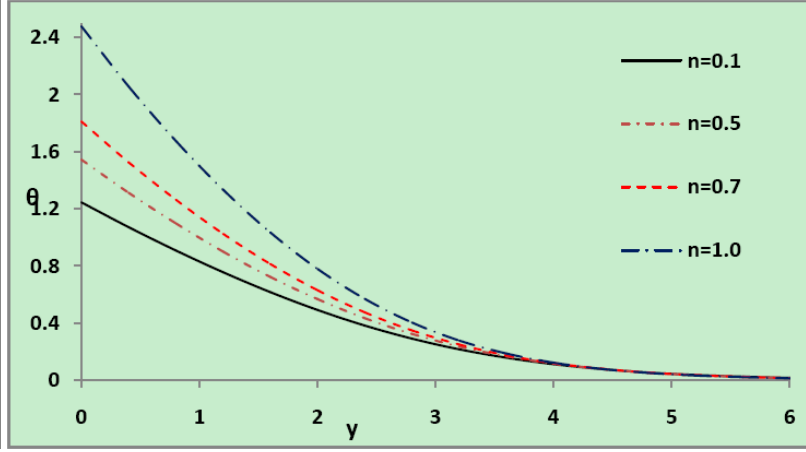

b)

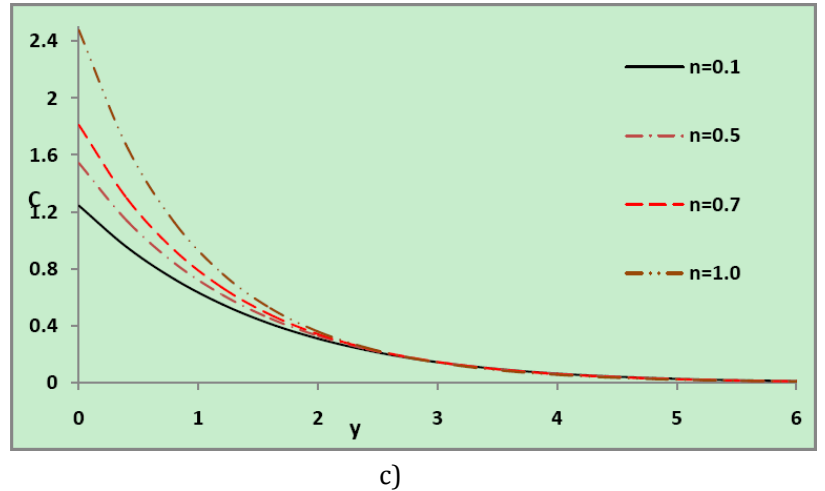

Fig. 2 a). Velocity profiles for different values of $n$; b) Temperature profiles for different values of $n$; c) Concentration profiles for different values of $n$.

Fig. 2 depicts the velocity, temperature and concentration profiles for different values of dimensionless exponential index $\mathrm{n}$. It can be seen that an increase in the values of $\mathrm{n}$ leads to an increase in the velocity distribution across the boundary layer. It is found that an increase in the values of $n$ leads to an increase in the temperature as well as concentration profiles.

The influence of Dufour number on velocity and temperature profiles are shown in Fig. 3.The Dufour number Du signifies the contribution of the concentration gradients to the thermal energy flux in the flow. It is noticed that the velocity and temperature profiles increases with the increase of Dufour number throughout the boundary layer.

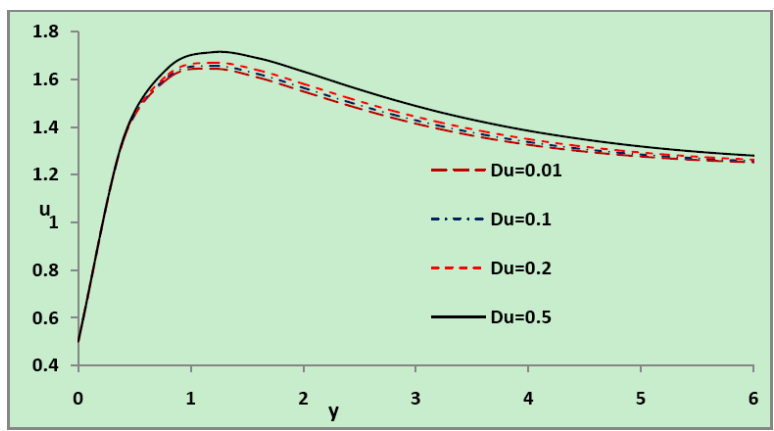

a)

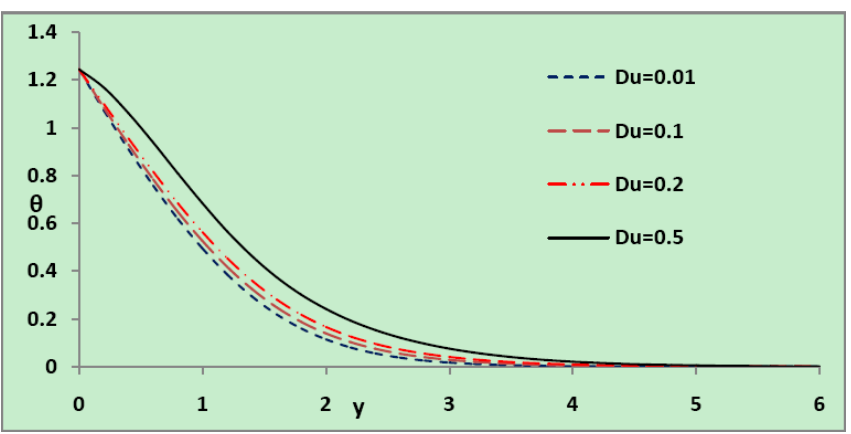

b)

Fig. 3 a). Velocity profiles for different values of Du; b) Temperature profiles for different values of Du.

The Fig. 4 shows that the effect of $S c$ on velocity and concentration profiles. The Schmidt number $S c$ embodies the ratio of the momentum to the mass diffusivity. The Schmidt number therefore quantifies the relative effectiveness of momentum and mass transport by diffusion in the hydrodynamic (velocity) and concentration (species) boundary layers. It can be seen that the velocity profile decreases with the effect 
of Sc. Also the concentration profile is decreases with increase of Sc. This causes the concentration buoyancy effects to decrease a yielding eradication in the fluid velocity.

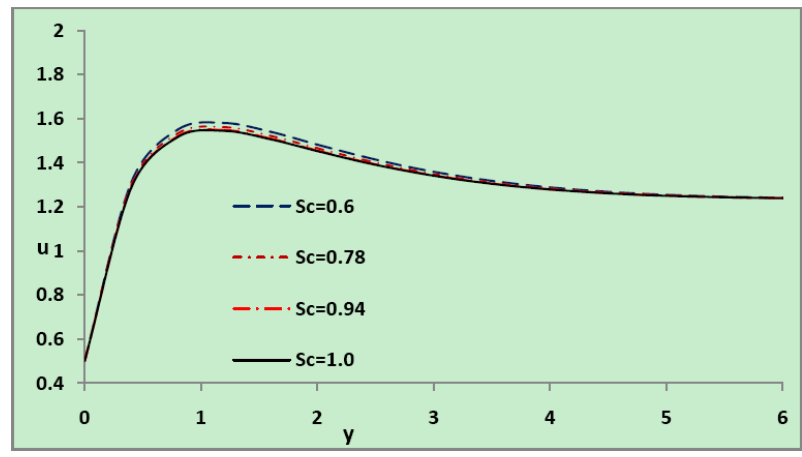

a)

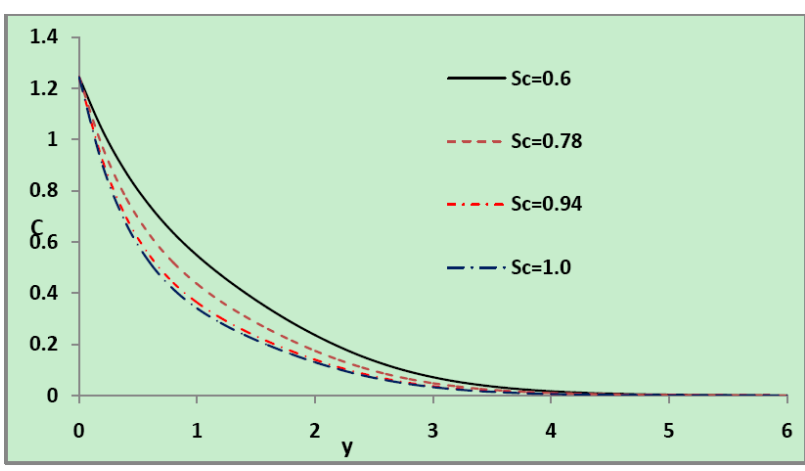

b)

Fig. 4 a). Velocity profiles for different values of $S c$; b) Concentration profiles for different values of $S c$.

The influence of Soret number on the velocity and concentration profiles is shown in Fig. 5. The Soret number $\mathrm{Sr}$ defines the effect of the temperature gradients inducing significant mass diffusion effects. It is found that the velocity and concentration profile increases with the increase of $\mathrm{Sr}$.

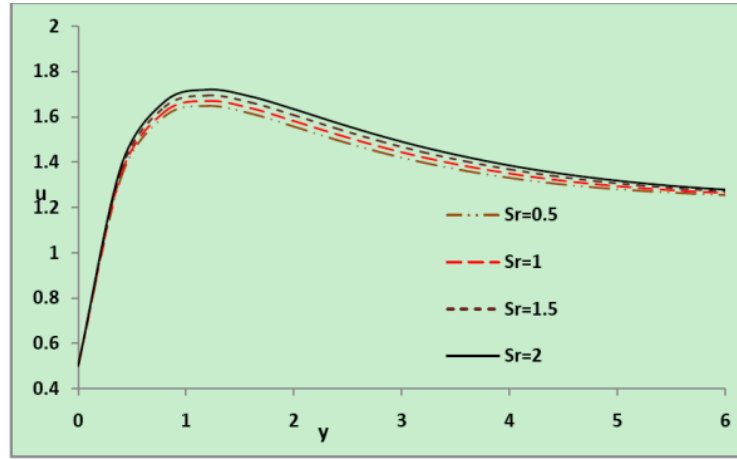

a)

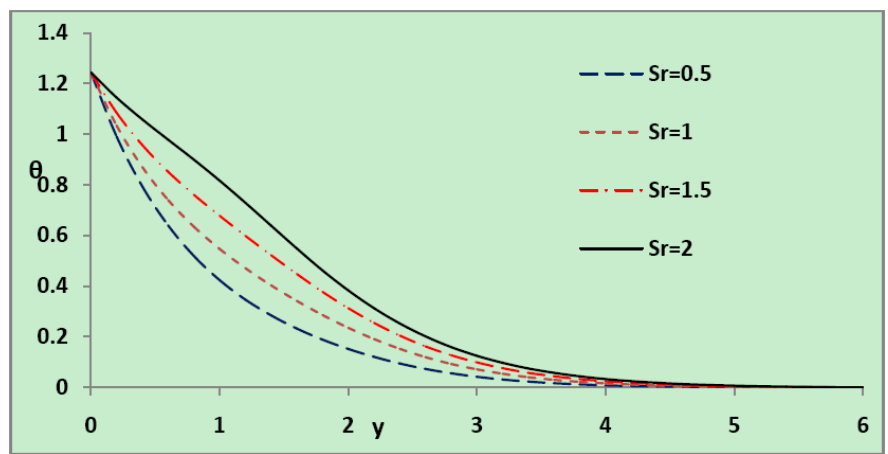

b)

Fig. 5 a). Velocity profiles for different values of $S r$; b) Concentration profiles for different values of $S r$.

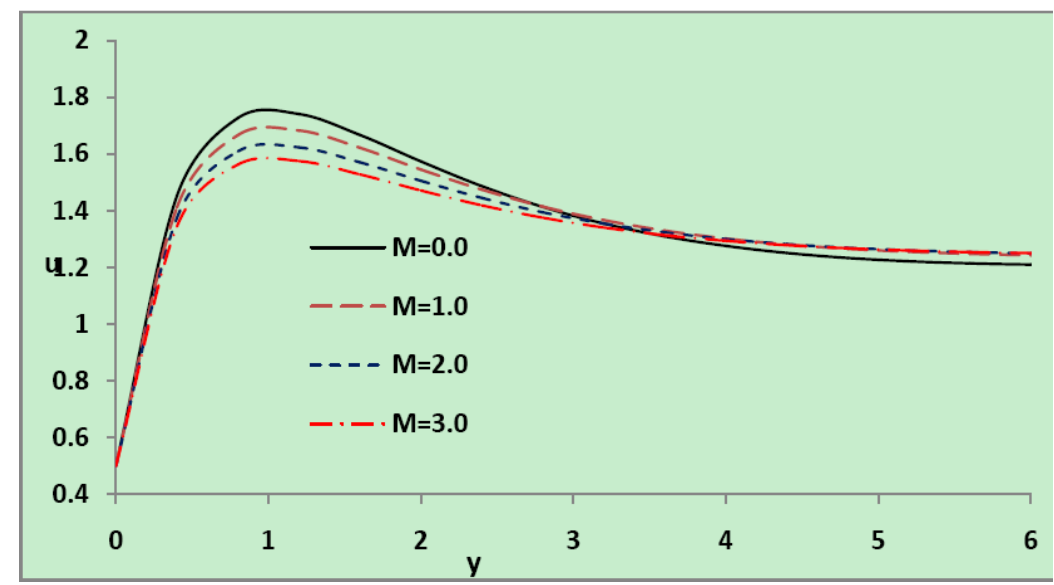

Fig. 6. Velocity profiles for different values of $M$.

The effect of magnetic field on the velocity profiles are plotted in Fig. 6. It can be seen that with the effect of magnetic field is to decelerate the velocity profiles. In Fig. 7 shows the velocity profile for different values of $\theta_{r}$. It is noticed from the figure that an increase in the value of viscosity variation parameter $\theta_{r}$ leads to an 
increase in the velocity profiles. Fig. 8 illustrates the variation of velocity distribution for different values of Up. It is found that for the different initial plate moving velocities, the velocity decreases to the constant value for the given material parameters .The effect of the given material parameters. The effect of the permeability of porous medium parameter $K$ is increasing the velocity with the increasing of the $K$ value is observed from Fig. 9.

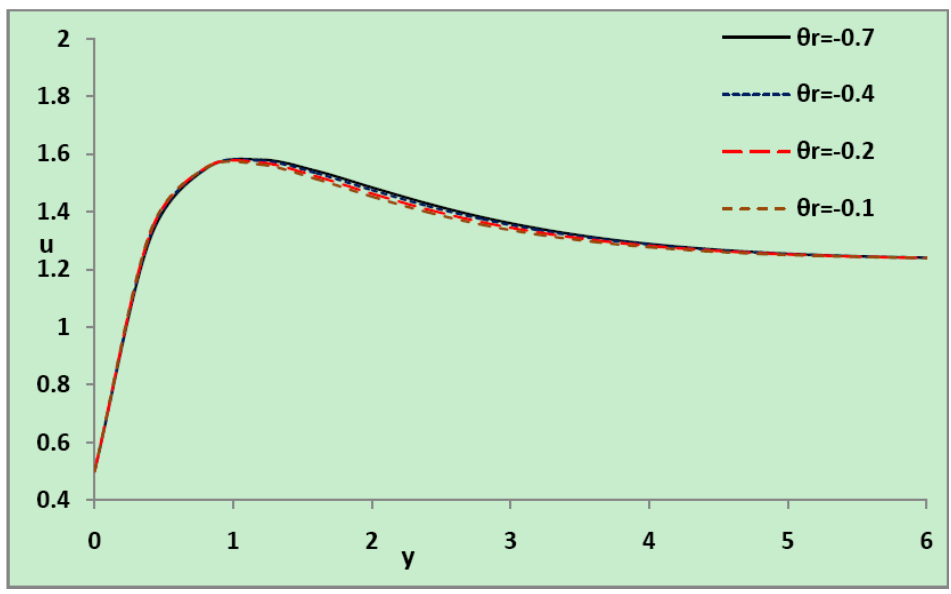

Fig. 7. Velocity profiles for different values of $\theta r$.

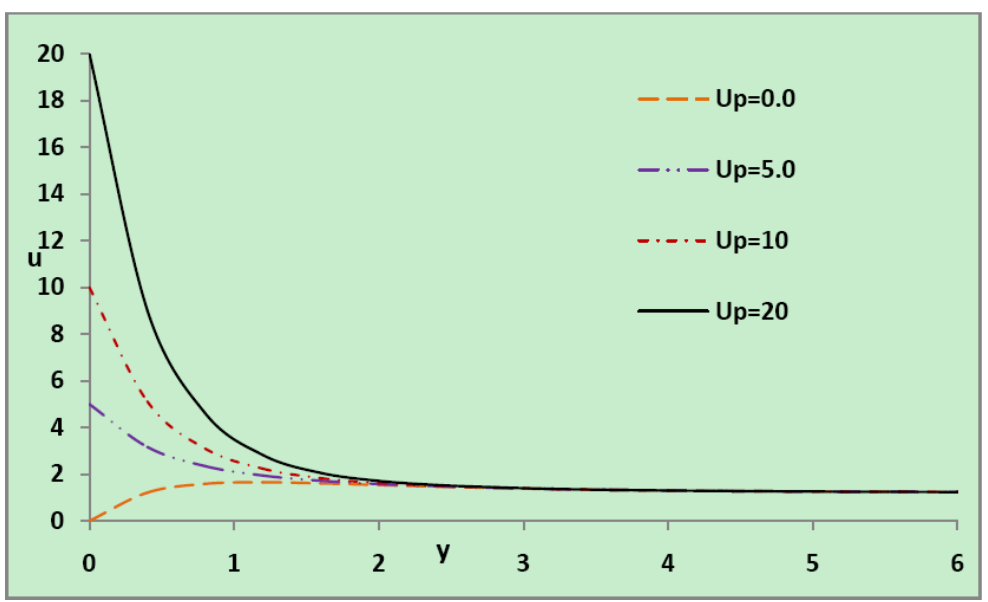

Fig. 8. Velocity profiles for different values of up.

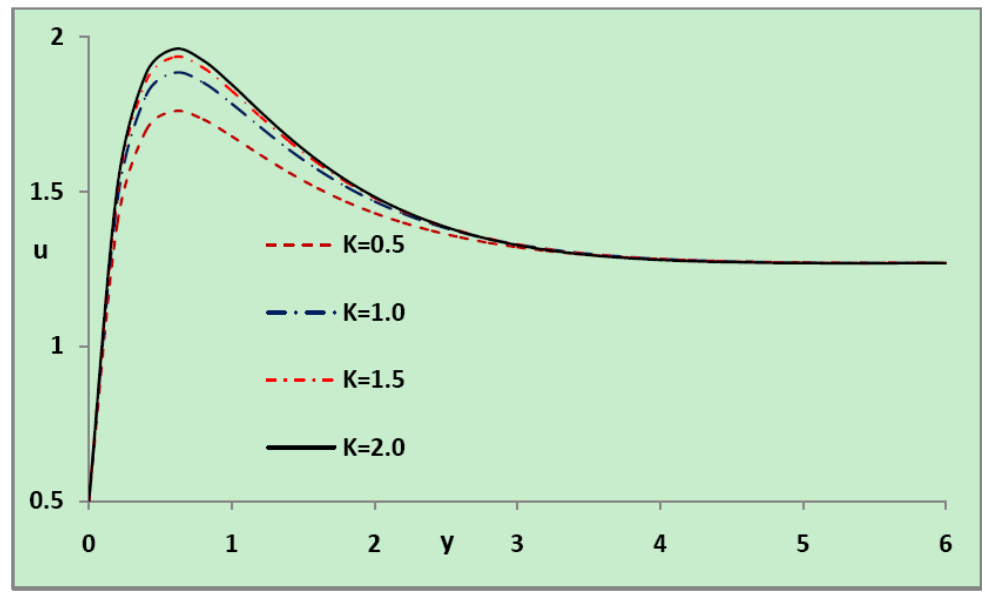

Fig. 9. Velocity profiles for different values of $K$.

Physically this result can be achieved when the holes of the porous medium may be neglected. For 
different values of the thermal conductivity parameter $\beta$ the temperature profile are plotted in Fig. 10. It is evident that from figure the temperature profiles increases with the increase of the thermal conductivity parameter $\beta$.

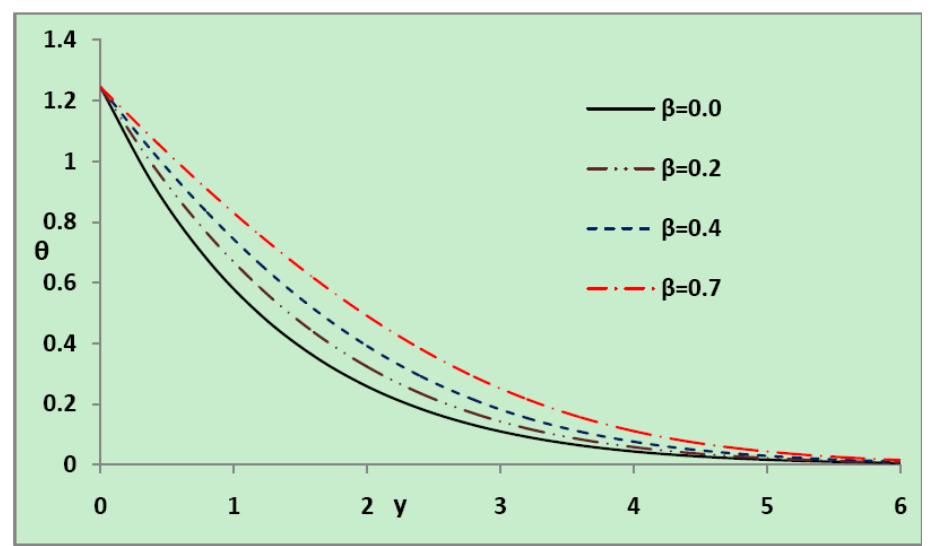

Fig. 10. Temperature profiles for different values of $\beta$.

Table 1. Effect of $G r, G m, M, K$ and on $C f$

\begin{tabular}{|l|l|l|l|l|l|}
\hline$G r$ & $G m$ & $M$ & $K$ & $\theta \mathrm{r}$ & $C_{f}$ \\
\hline 2 & 2 & 1 & 0.5 & -0.6 & 3.6946 \\
\hline 4 & 4 & 1 & 0.5 & -0.6 & 5.044773 \\
\hline 2 & 2 & 2 & 0.5 & -0.6 & 3.561448 \\
\hline 2 & 2 & 1 & 1 & -0.6 & 3.857552 \\
\hline 2 & 2 & 1 & 0.5 & -0.2 & 3.733612 \\
\hline
\end{tabular}

Table 1 shows the effects of thermal Grashof Number Gr, solutal Grashof Number Gm, magnetic field parameter $M$, porous medium parameter $K$, and variable viscosity parameter $\theta r$ on the local skin friction coefficient $C_{f}$. It is observed that as $G r$ and $G m$ or $K$ or $\theta r$ increases the skin friction coefficient increases.

Table 2. Effect of $\beta$ on $C_{f}$ and $N u \mathrm{Re}_{x}^{-1}$

\begin{tabular}{|l|l|l|}
\hline $\mathrm{B}$ & $C_{f}$ & $N u \mathrm{Re}_{x}^{-1}$ \\
\hline 0 & 3.72571 & 1.565111 \\
\hline 0.2 & 3.721877 & 1.242303 \\
\hline 0.4 & 3.711814 & 1.001896 \\
\hline 0.7 & 3.697474 & 0.760436 \\
\hline
\end{tabular}

From Table 2 it is noticed that with the increase of thermal conductivity parameter $\beta$ the skin friction coefficient increases and decreases the Nusselt number.

Table 3. Effect of $S c$ on $C_{f}$ and $S h \mathrm{Re}_{x}^{-1}$

\begin{tabular}{|l|l|l|}
\hline$S c$ & $C_{f}$ & $S h \mathrm{Re}_{x}^{-1}$ \\
\hline 0.6 & 3.6946 & 1.052415 \\
\hline 0.78 & 3.612298 & 1.378964 \\
\hline 0.94 & 3.554511 & 1.640368 \\
\hline 1 & 3.53714 & 1.731945 \\
\hline
\end{tabular}

Table 3 display the values of $C_{f}$ and the coefficient of Sherwood number Sh with the increase of $S c$. The $C_{f}$ decreases an increases the Sherwood number. 


\section{Conclusions}

A comprehensive numerical parametric study for the numerical solutions of a class of nonlinear equations is conducted and results are reported in terms of graphs. This is done in order to illustrate special features of the solutions. So the numerical solutions by using finite difference method were obtained. Apart from that, obtained results indicate that it is an adequate scheme for the solution of the present problems. From the present numerical study the following conclusions can be drawn

1) The velocity and temperature profile increases with the increase of Dufour number throughout the boundary layer.

2) The Schmidt number quantifies the relative effectiveness of momentum and mass transport by diffusion in the hydrodynamic and concentration boundary layers.

3) With the increase of thermal conductivity parameter $\beta$ the skin friction coefficient increases and decreases the Nusselt number.

4) The temperature profiles increases with the increase of the thermal conductivity parameter $\beta$.

5) Velocity distribution attains a distinctive maximum value in the vicinity of the plate surface and then decreases properly to approach the free stream value.

\section{References}

[1] Kaviancy, M. (1992). Principles of Heat Transfer in Porous Media. New-York: McGraw-Hill.

[2] Dunn, J. C., \& Hardee, H. C. (1981). Jvolcanology and Geothermal Res., 11, 189.

[3] Nield, D. A., and Bejan, A. (2006). Convection in Porous Media (3 $3^{\text {rd }}$ ed.). New-York: Springer.

[4] Bejan, A., \& Khair, K. R. (1985). Int J. Heat Mass Transfer, 28, 909.

[5] Lai, F. C., \& Kulacki, F. A. (1991). Coupled heat and mass transfer by natural convection from vertical surfaces in a porous medium. Int. J Heat Mass Transfer, 34, 1189-1194.

[6] Vafai, K. (2000). Handbook of Porous Media. New York: Marcel Dekker.

[7] Ingham, D., \& Pop, I. Transport Phenomena in Porous Media II.

[8] Raptis, A., Massalas, C., \& Tzivanids, G. (1982). Hydromagnetic free convection flow through a porous medium between two parallel plates. Phys Lett., 90A, 288-289.

[9] Gribben, J. (1965). The magneto hydrodynamic boundary layer in the presence of a pressure gradient. Proceedings of Royal Soc. London, 287, (pp. 123-141).

[10] Raptis, A. (1986). Flow through a porous medium in the presence of magnetic field. Int. J Energy Res., 10, 97-101.

[11] Helmy, K. A. (1998). MHD Unsteady Free Convection Flow Past a Vertical Porous Plate, 78, 255-270.

[12] Youn, J. K. (2000). Unsteady MHD convective heat transfer past semi-infinite vertical porous moving plate with variable suction. Int. J. Eng. Sci., 38, 833-845.

[13] Hossain, M., \& Munir, S. (2000). Mixed convection flow from a vertical plate with temperature dependent viscosity. Int. J. Therm. Sci., 39, 173-183.

[14] Hossain, M., Khanafer, K., \& Vafai, K. (2001). The effect of radiation on free convection flow of fluid with variable viscosity from porous vertical plate. Int. J. Therm. Sci., 40, 115-124.

[15] Eckert, E. R. G., \& Drake, R. M. (1972). Analysis of Heat and Mass Transfer. New York: McGraw-Hill.

[16] Jha, B. K., \& Singh, A. K. Soret effects on free convection and mass transfer flow in stokes problem for an infinite vertical plate. Astrophys.Space Sci., 173, 251-255.

[17] Kishan, N., Reddy, M. C. K., \& Govardhan, K. (2009). MHD free convection heat and mass transfer in a doubly sratified Darcy porous medium considering soret and dufour effects with viscous dissipation. Int. J. of Applied mechanics and Engineering, 14(3), 733-745. 
[18] Gnaneswara, R. M., \& Bhasker, R. N. (2009). Unsteady MHD convective heat and mass transfer past a semi-infinite vertical porous plate with variable viscosity and thermal conductivity. International Journal of Applied Mathematics and computation, 1(2), 10.

Deepa Gadipally was born and brought up in Hyderabad District, Telangana State. She obatined her B.Sc, M.Sc and Ph.d degrees from Osmania University. She is working as an assistant professor in mathematics Department in C.B.I.T. Her research interests are in convection flows, heat and mass transfer problems.

Murali Gundagani was born and brought up in Nalgonda District, Telangana State. He obatined his Ph.d degree from Allahabad University of Science and Agriculture (SHIATS) and completed his B.Sc, M.Sc degrees from Osmania University. He is working as a coordinator in Mathematics Department in GITAM University. His research interests are in convection flows, heat and mass transfer problems. 